Pak. J. Agri. Sci., Vol. 53(1), 35-41; 2016

ISSN (Print) 0552-9034, ISSN (Online) 2076-0906

DOI: 10.21162/PAKJAS/16.4441

http://www.pakjas.com.pk

\title{
METHYL JASMONATE BRINGS ABOUT RESISTANCE AGAINST SALINITY STRESSED TOMATO PLANTS BY ALTERING BIOCHEMICAL AND PHYSIOLOGICAL PROCESSES
}

\author{
Abdul Manan ${ }^{1, *}$, C.M. Ayyub ${ }^{1}$, M. Aslam Pervez ${ }^{1}$ and Rashid Ahmad ${ }^{2}$ \\ ${ }^{1}$ Institute of Horticultural Sciences, University of Agriculture, Faisalabad, Pakistan; ${ }^{2}$ Department of Agronomy, \\ University of Agriculture, Faisalabad, Pakistan. \\ *Corresponding author's e-mail: mananbukhari@gmail.com
}

\begin{abstract}
This manuscript mainly defines about the effect of foliar application of methyl jasmonate $\left(\mathrm{C}_{13} \mathrm{H}_{20} \mathrm{O}_{3}\right)(\mathrm{MeJA})$ on physiological and biochemical processes in tomato under both saline and non-saline conditions. Two tomato genotypes Rio Grande (tolerant) and Savera (sensitive) were grown in pots having sand as growth medium. The salinity substantially decreased the physiological and biochemical parameters. Different doses of MeJA $(0.0,10,20,30,40,50,60 \mu \mathrm{M})$ were applied on both control and salt stressed tomato plants. Methyl Jasmonate MeJA significantly ameliorated the deleterious effects of salinity on tomato plants by inducing the physiological and biochemical resistance. Different parameters responded to MeJA at various extents. Our findings illustrate that all the parameters responded to foliar application of MeJA and it is quite helpful creating physiological and biochemical resistance in salinity stressed tomato plants.
\end{abstract}

Keywords: Tomato, methyl jasmonate, photosynthesis, transpiration, antioxidants, proline, amino acids.

\section{INTRODUCTION}

Abiotic stresses are likely to be common due to climate change being emerging threat of $21^{\text {st }}$ century in past decades to crop productivity. Drought stress heat stress, salinity stress and other abiotic stresses are soaring complexities to crop husbandry. Changing cropping pattern, irrigation with substandard water and soil deterioration finally lead to effect crop productivity (Nawaz et al., 2015). Out of abiotic stresses salt stress has become an acute problem to farm land and productivity. Salt affected soils have large quantities of $\mathrm{Na}^{+}$and $\mathrm{Cl}^{-}$ions that hamper the plant physiological and biochemical processes, ultimately exert abiotic stress to plant (Munns and Tester, 2008). Overall 7\% of total earth surface making 800 million hectares of land and $20 \%$ of irrigated farmland is exposed to salinity (Aoki et al., 2005; Flowers and Yeo, 1995; Munns and Tester, 2008).

Phytohormones have been reported to cope stress in plants by producing proteins and causing resistance like abscisic acid (Jin et al., 2000), salicylic acid (Poor et al., 2011) and methyl jasmonate (MeJA) (Yoon et al., 2009). MeJA has been reported to attenuate the drastic effects of salinity and water stress in different crop plants like soybean (Anjum et al., 2011; Yoon et al., 2009), barley (Tsonev et al., 1998), strawberry (Wang, 1999), pea (Velitchkova and Fedina, 1998) and broccoli (Del Amor and Cuadra-Crespo, 2011).

Jasmonic acid (JA) and its methyl ester methyl jasmonate (MeJA) collectively termed as jasmonates are reported to cause cell signaling and regulatory phenomenon responsible to affect seed germination, tuberization, senescence, root growth, reproductive growth and fruit ripening (Creelman and Mulpuri, 2002; Wasternack and Hause, 2002). MeJA under control conditions reduces gaseous exchange parameters by stomatal closure (Suhita et al., 2003; Wang, 1999) facilitated by alkalization of the guard cell (Gehring $e t$ al., 1997) but under stress conditions reveals significant improvement in physiological and biochemical processes (Popova et al., 2003).

Tomato is a popular annual vegetable crop consumed fresh, cooked or processed: by various ways into various products. Deleterious effects of salinity on physiology and biochemistry of tomato is well documented (Cuartero and Fernandez-Munoz, 1998; Maggio et al., 2007; RomeroAranda et al., 2001). In this way this research project was designed to check the possible effects of MeJA on salt stressed tomato plants. One of the key objectives of this study was to optimize the best dose of MeJA that can recover the most from the drastic effects of salinity on tomato.

\section{MATERIALS AND METHODS}

Plant material and growth condition: Present study was carried out in Institute of Horticultural Sciences (IHS), University of Agriculture, Faisalabad. Seeds of tomato were obtained from Ayub Agriculture Research Institute (AARI) Faisalabad. Seeds of tomato genotypes Rio Grande and Savera were surface sterilized before sowing with $5 \%$ sodium hypochlorite for ten minutes and then rinsed with pure water. Ten seeds were then sown in each sand filled 
plastic pot (9 L volume) then thinned to five per pot. Half strength Hoagland solution was used as source of nutrition in the sand. There were three pots in each treatment and each pot represented as one experimental unit. Leaf samples and other parameters were taken at seedling stage (50 days after sowing). Thirty days after sowing the salinity treatments were applied and after one week MeJA treatments were applied as foliar application method.

Determination of gaseous exchange parameters; photosynthesis $(A)$, transpiration $(E)$ and stomatal conductance (gs): For the measurement of physiological attributes such as photosynthesis rate (A), transpiration rate (E) and stomatal conductance (gs) we selected the three young fully developed and healthy leaves. These selected leaves were placed one by one in the chamber of portable apparatus termed as Infra-Red Gas Analyzer (IRGA) (Analytical Development Company, Hoddesdon, England).

Determination of chlorophyll contents: In this study, we calculated the chlorophyll contents by following the method of Arnon (1949) and Davies (1976). Fresh leaves were cut in minute segments and one gram of these minute segments was put into small sterilized plastic bottles, having $80 \%$ acetone solution. These plastic bottles were allowed to stay at room temperature overnight. The extract obtained from the plastic bottles was centrifuged at $14000 \mathrm{x} g$ for 5 minutes and the supernatant thus obtained was taken at absorbance 645, 663 and $480 \mathrm{~nm}$, using double beam Spectrophotometer (Hitachi-120, Japan).

$\mathrm{Chl} \mathrm{a}=[12.7(\mathrm{OD} 663)-2.69(\mathrm{OD} 645)] \times \mathrm{V} / 1000 \times \mathrm{W}$

$\mathrm{Chl} \mathrm{b}=[22.9(\mathrm{OD} 645)-4.68(\mathrm{OD} 663)] \times \mathrm{V} / 1000 \times \mathrm{W}$

Where $\mathrm{V}=$ Volume of extract and $\mathrm{W}=$ Weight of sample

Determination of total free amino acids (TFA) and total soluble protein (TSP) and leaf proline contents: Fresh leaf material of weight $0.5 \mathrm{~g}$ was taken and TFA, TSP and leaf proline contents were estimated by the protocol of Hamilton and Van Slyke, (1943), Lowry et al. (1951) and Bates et al. (1973), respectively.

Assay of catalase and peroxidase activity: Catalase (CAT) and peroxidase (POX) activities were measured by the procedure of Chance and Maehly (1955) with some alteration. The CAT reaction solution $(3 \mathrm{~mL})$ was comprised of $50 \mathrm{mM}$ phosphate buffer $(\mathrm{pH} 7.0), 5.9 \mathrm{mM} \mathrm{H}_{2} \mathrm{O}_{2}$ and 0.1 $\mathrm{mL}$ of enzyme extract. Changes in absorbance of the reaction solution were recorded after every $20 \mathrm{~s}$ at $240 \mathrm{~nm}$. One unit CAT activity was specified as an absorbance change of 0.01 units per min. The POX reaction solution (3 $\mathrm{mL}$ ) was comprised of $50 \mathrm{mM}$ phosphate buffer ( $\mathrm{pH} 5.0), 20$ $\mathrm{mM}$ guaiacol, $40 \mathrm{mM} \mathrm{H} \mathrm{H}_{2}$ and $0.1 \mathrm{~mL}$ of enzyme extract. Variations in absorbance of the reaction solution at $470 \mathrm{~nm}$ were calculated after every 20 seconds. One unit POX activity was assigned as an absorbance change of 0.01 units per min. The POX activity was determined and expressed as unit $\min ^{-1} \mathrm{~g}^{-1} \mathrm{FW}$ basis.

Statistical analysis and design: All the treatments were arranged in CRD (completely randomized design). Data recorded were subjected to statistical analysis by analysis of variance technique using STATISTIX computer program. LSD at $5 \%$ level of probability was used to compare the individual means.

\section{RESULTS}

Gaseous exchange attributes: Photosynthesis rate in both tomato genotypes reduced markedly due to the imposition of salt stress. However, the deleterious effect of salinity was more pronounced on 'Savera', as compared to 'Rio Grande'. Overall, MeJA application resulted in 1.08-fold higher photosynthesis rate in salt tolerant genotype 'Rio Grande', as compared to 'Savera' under saline as well as non-saline conditions. Foliar spray of MeJA enhanced the photosynthesis rate under salt stressed but reduced under non-stressed plants (Fig. 1). Under non-saline conditions, MeJA caused the decrease in photosynthesis rate for salt tolerant genotype (Rio Grande) but minimum was recorded for savera $\left(8.95 \mu \mathrm{mol} \mathrm{CO}_{2} \mathrm{~m}^{-2} \mathrm{~s}^{-1}\right)$. Overall, exogenous application of 50 micro molar MeJA was more helpful for attaining salt tolerance in tomato.

Transpiration rate was decreased in both tested genotypes

Table 1. Summary of ANOVA table of photosynthesis rate (A), transpiration rate (E), stomatal conductance (gs), chlorophyll contents "a" and " $b$ ", total soluble proteins (TSP), total free amino acid (TFA), catalase (CAT) and peroxidase (POX).

\begin{tabular}{|c|c|c|c|c|c|c|c|c|c|c|}
\hline SOV & $\mathbf{A}$ & $\mathbf{E}$ & gs & Chlorophyll "a" & Chlorophyll "b" & Proline contents & TSP & TFA & CAT & POX \\
\hline$\overline{\operatorname{MeJA}(\mathrm{M})}$ & $* * *$ & $* * *$ & $* * *$ & $* * *$ & $* * *$ & $* * *$ & $* * *$ & $* * *$ & $* * *$ & $* * *$ \\
\hline Salinity (S) & $* * *$ & $* * *$ & $* * *$ & $* * *$ & $* * *$ & $* * *$ & $* * *$ & $* * *$ & $* * *$ & $* * *$ \\
\hline Genotypes (G) & $* * *$ & $* * *$ & $* * *$ & $* * *$ & $* * *$ & $* * *$ & $* * *$ & $* * *$ & $* * *$ & $* * *$ \\
\hline $\mathrm{M} \times \mathrm{S}$ & NS & NS & NS & $* * *$ & $*$ & NS & NS & $* * *$ & NS & NS \\
\hline $\mathrm{M} \times \mathrm{G}$ & NS & NS & NS & $* * *$ & NS & NS & NS & NS & NS & NS \\
\hline $\mathrm{S} \times \mathrm{G}$ & NS & NS & NS & $* * *$ & NS & NS & NS & NS & NS & NS \\
\hline $\mathrm{M} \times \mathrm{S} \times \mathrm{G}$ & NS & NS & NS & $* * *$ & NS & NS & NS & NS & NS & NS \\
\hline $\mathrm{CV}$ & 2.89 & 11.11 & 6.55 & 4.41 & 8.57 & 5.98 & 3.84 & 5.60 & 2.11 & 2.01 \\
\hline
\end{tabular}


under salt stressed conditions. The plants grown under nonsaline conditions exhibited the highest transpiration rate than those grown under saline conditions. Under saline condition, highest value for transpiration rate was observed in $50 \mu \mathrm{M}$ MeJA-treated plants showing about 1.01-fold increase in transpiration, as compared to control plants in saline as well as non-saline conditions, respectively. Furthermore, 'Rio Grande' genotype exhibited significantly about 1.34-fold higher transpiration rate, as compared to 'Savera'. However, under saline as well as non-saline conditions 'Rio Grande' showed 1.40- and 1.29-fold higher transpiration rate, respectively, as compared to 'Savera' genotype (Fig. 2).

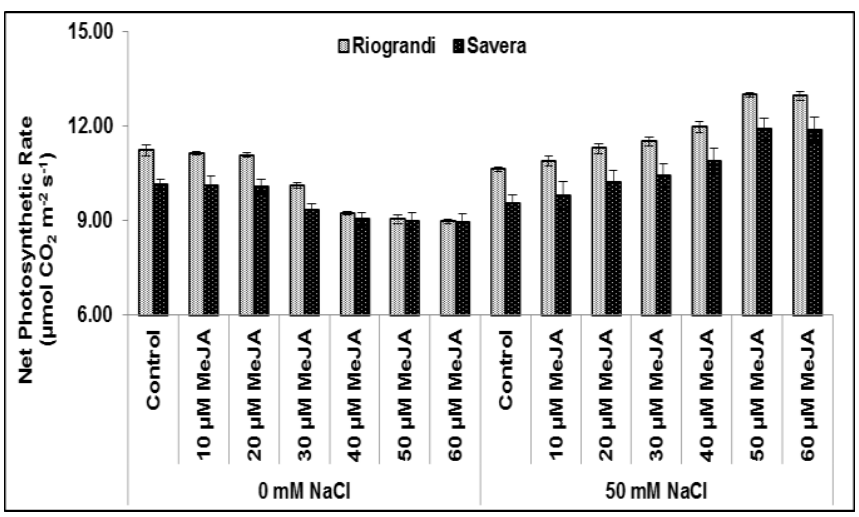

Figure 1. Effect of salinity stress and MeJA on net photosynthetic rate (A). All values are mean of three replications, vertical bars are $\pm \mathrm{SE}$.

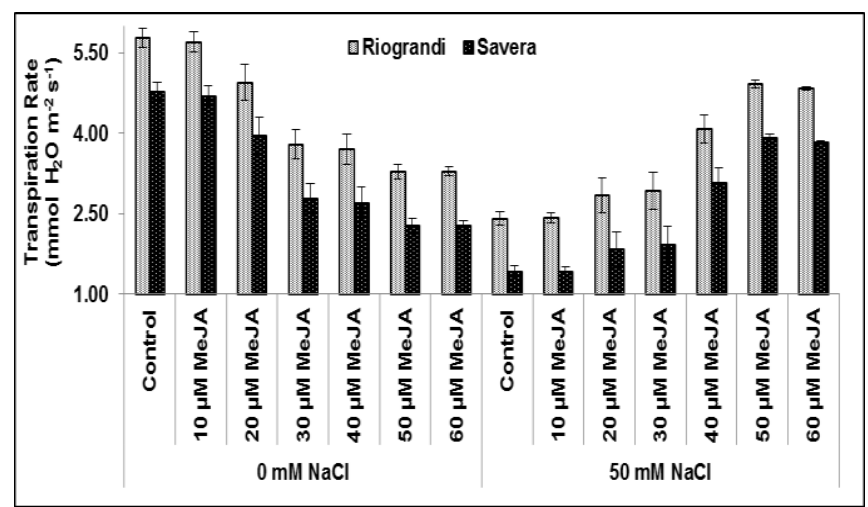

Figure 2. Effect of salinity stress and MeJA on transpiration rate $(\mathbf{E})$. All values are mean of three replications, vertical bars are $\pm \mathrm{SE}$.

Sodium chloride in growth medium reduced the stomatal conductance in both genotypes. Exogenous application of MeJA substantially enhanced stomatal conductance under saline but reduced under non-saline conditions. Mean stomatal conductance values of both genotypes observed in $50 \mu \mathrm{M}$ MeJA-treated plants was about 1.18- and 1.19-fold higher than control under saline conditions; whereas, stomatal conductance under non-saline condition was about 1.06-fold higher than saline conditions. Overall, salt tolerant genotype 'Rio Grande' exhibited about 1.11-fold higher stomatal conductance, as compared to 'Savera' (Fig. 3).

Chlorophyll contents: Chlorophyll "a" in both tomato genotypes reduced markedly due to the imposition of salt stress (Fig. 4). Deleterious effect of salinity was more in 'Savera', as compared to that on Rio Grande. Overall, chlorophyll "a" contents were 1.17 -fold more in 'Rio Grande', as compared to 'Savera' genotype. Foliar spray of $60 \mu \mathrm{M}$ MeJA enhanced chlorophyll "a" contents upto 1.37fold than control plants under salt stressed conditions. Under saline conditions both tomato genotypes positively responded to the MeJA application; however, 'Rio Grande' showed maximum increase in chlorophyll "b" contents, as compared to 'Savera'. Foliar application of MeJA on tomato plants showed increase in chlorophyll "b" contents under salt stress; whereas, chlorophyll "b" contents were continuously decreased in non-saline conditions, despite MeJA application. Overall, 'Rio Grande' exhibited significantly about 1.36-fold higher chlorophyll "b" contents, than 'Savera' genotype under both soil conditions (Fig. 5).

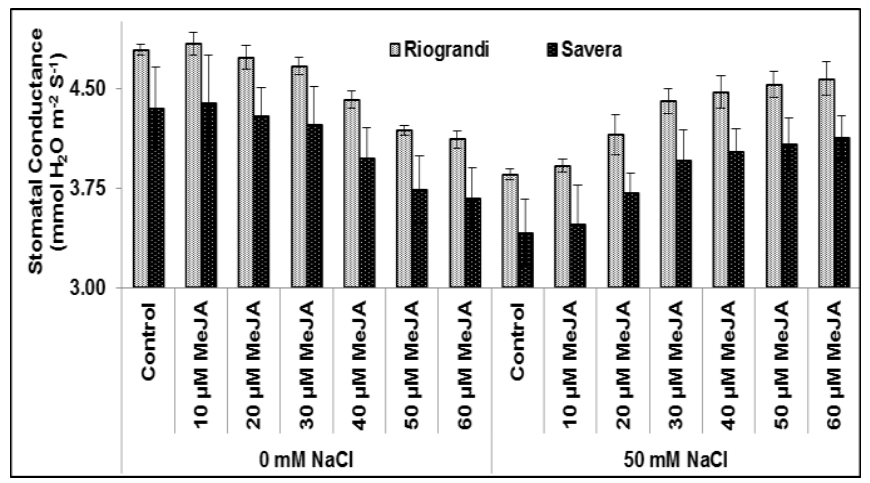

Figure 3. Effect of salinity stress and MeJA on stomatal conductance (gs). All values are mean of three replications, vertical bars are $\pm \mathrm{SE}$.

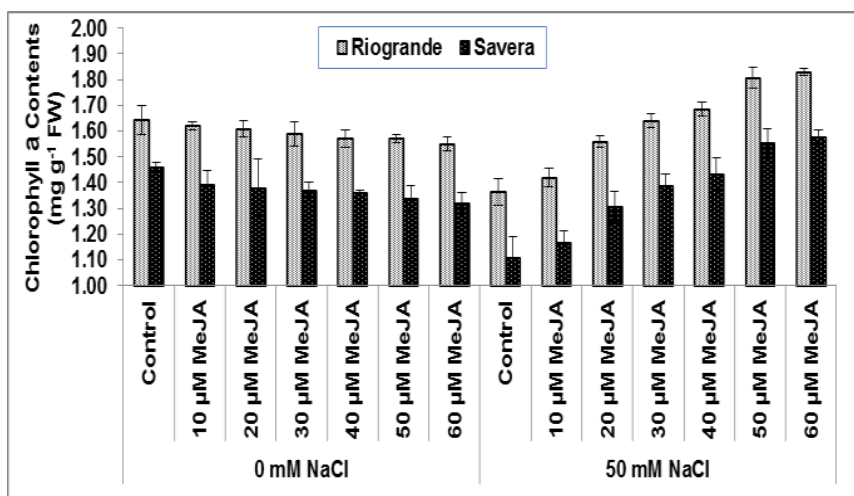

Figure 4. Effect of salinity stress and MeJA on chlorophyll "a" contents. All values are mean of three replications, vertical bars are $\pm \mathrm{SE}$. 


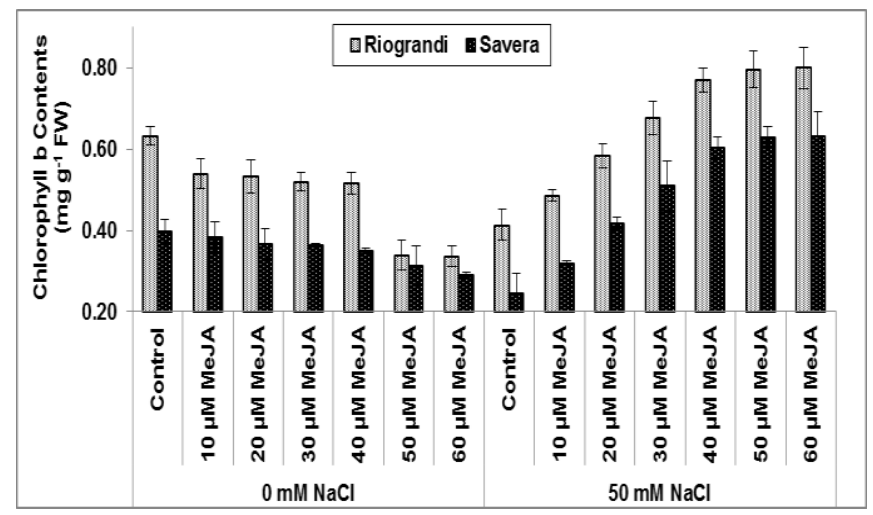

Figure 5. Effect of salinity stress and MeJA on chlorophyll "b" contents. All values are mean of three replications, vertical bars are \pm SE.

Proline contents: Proline contents were significantly higher 2.12-fold under salt stressed conditions due to application of MeJA, as compared to non-saline conditions. Under nonsaline conditions, $50 \mu \mathrm{M}$ MeJA resulted in 1.68-fold higher proline contents; whereas, under salt stressed conditions 1.26-fold higher proline contents were observed in $50 \mu \mathrm{M}$ treated tomato plants, as compared to control. Overall, salt tolerant genotype 'Rio Grande' exhibited 1.18-fold higher proline contents than 'Savera' genotype (Fig. 6).

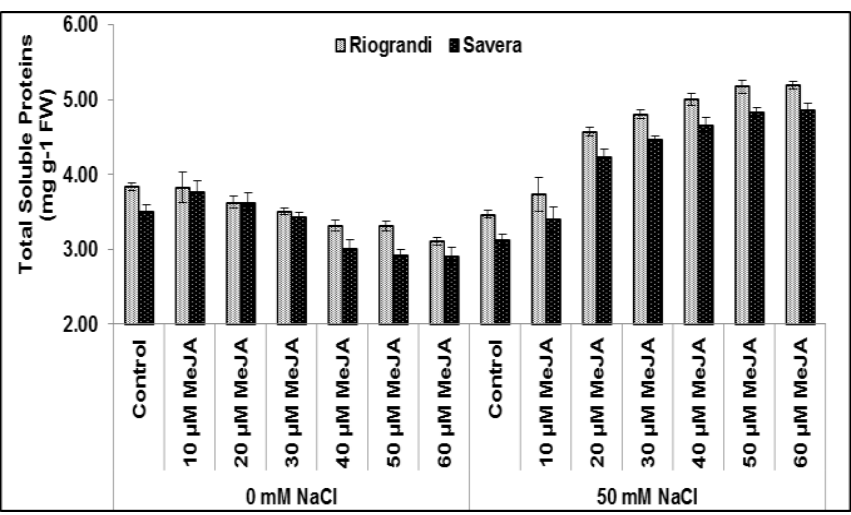

Figure 6. Effect of salinity stress and MeJA on total soluble proteins (TSP). All values are mean of three replications, vertical bars are \pm SE.

Total soluble proteins (TSP): Overall Rio Grande produced 1.07 fold more TSP than Savera both under saline and normal conditions. MeJA revealed a significant effect of TSP, foliar application of MeJA produced more than $66 \%$ TSP than control. Under non-saline conditions TSP decreased but under saline conditions TSP increased with MeJA application (Fig. 7).

Total free amino acids (TFA): A distinct change was observed in amino acid contents of the tomato genotypes, investigated under stressed and non-stressed conditions. Application of MeJA enhanced the level of amino acid in salt affected soil about 3.02-fold higher than non-stressed conditions. Moreover, salt tolerant 'Rio Grande' genotype exhibited 1.14-fold higher amino acid contents than 'Savera'. Exogenous application of $60 \mu \mathrm{M}$ MeJA resulted in 1.80- and 2.39-fold higher amino acids than control tomato plants in non-saline as well as saline conditions, respectively (Fig. 8).

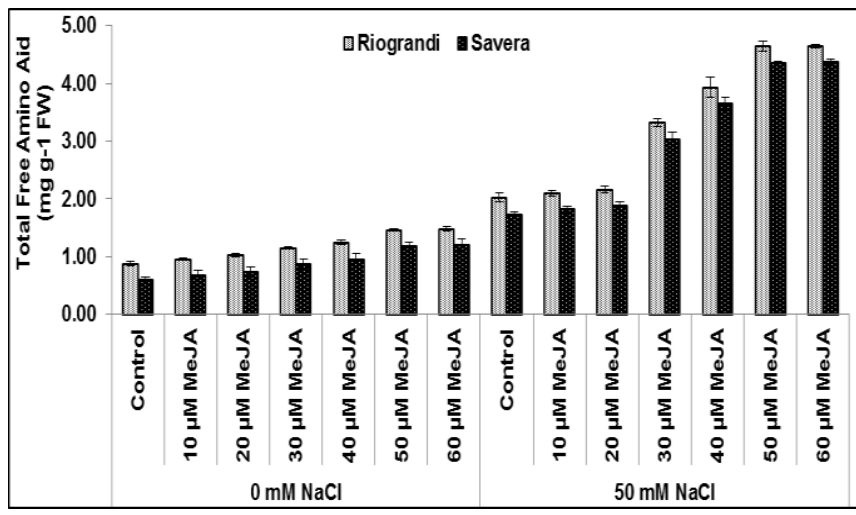

Figure 7. Effect of salinity stress and MeJA on total free amino acids (TFA). All values are mean of three replications, vertical bars are \pm SE.

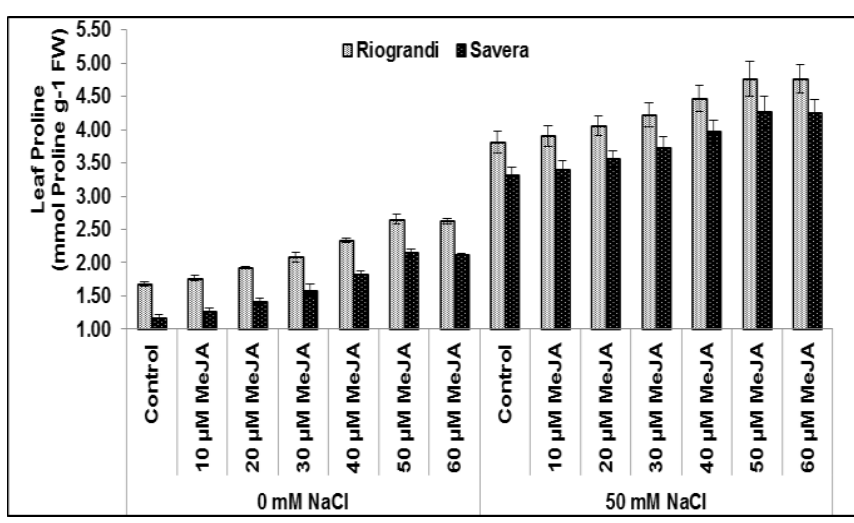

Figure 8. Effect of salinity stress and MeJA on leaf proline contents. All values are mean of three replications, vertical bars are \pm SE.

Antioxidants enzymatic: POX enzyme activity was gradually increased with increasing concentration of MeJA under non-saline and saline conditions. However, increase in POX activity was 1.07 -fold more in saline conditions, as compared to non-saline conditions; whereas, salt tolerant genotype 'Rio Grande' exhibited about 1.05-fold increase in POX enzyme activity than 'Savera'. Lowest activity of POX enzyme was observed in control plants, showing about 1.24and 1.26-fold less POX enzyme activity under non-saline as well as saline condition, respectively, as compared to $60 \mu \mathrm{M}$ MeJA-treated tomato plants (Fig. 9).

In present study, salt stress increased the enzymatic activity of catalase (CAT) in the tested genotypes. Salt tolerant 
genotype of tomato 'Rio Grande' exhibited significantly about 1.06-fold higher activity than 'Savera'. Whereas; application of MeJA resulted 1.09-fold higher CAT activity in saline conditions, as compared to non-saline conditions. Furthermore, CAT activity was 1.32- and 1.29-fold higher in $60 \mu \mathrm{M}$ MeJA-treated tomato plants in non-saline as well as saline condition, as compared to control (Fig. 10).

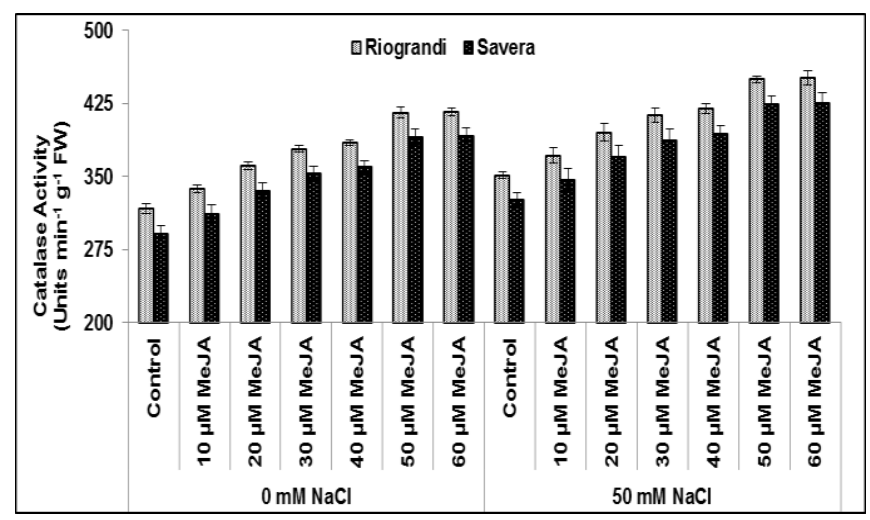

Figure 9. Effect of salinity stress and MeJA on catalase activity (CAT). All values are mean of three replications, vertical bars are $\pm \mathrm{SE}$.

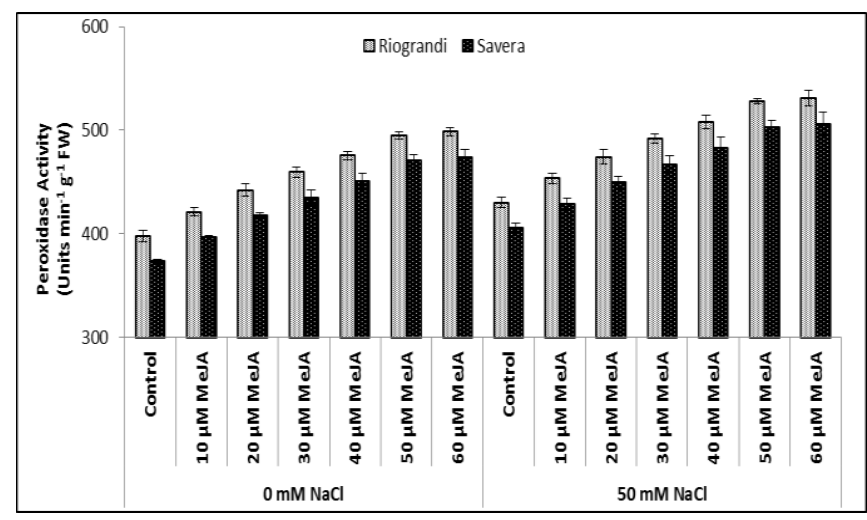

Figure 10. Effect of salinity stress and MeJA on catalase activity (CAT). All values are mean of three replications, vertical bars are $\pm \mathrm{SE}$.

\section{DISCUSSION}

Gaseous exchange: Salinity significantly decreased photosynthesis rate (A) and other gaseous exchange parameters by the osmotic effect of salinity that leads to ABA accumulation (Jia et al., 2002) that further leads to stomatal closure thus interrupts stomatal conductance and transpiration (Garcia-Mata and Lamattina, 2002), loss of pigments, activity of Rubisco (Lutts et al., 1996). Under non-saline conditions MeJA application lead to reduction in gaseous exchange parameters but under salt stress conditions MeJA significantly recovered the photosynthesis rate. This could be due to the production of protective protein and proper working of photosynthesis machinery (Velitchkova and Fedina, 1998; Yoon et al., 2009). MeJA induced enhanced production of chlorophyll contents can be another reason under salt stress conditions for increased photosynthetic rate (Yoon et al., 2009).

Chlorophyll contents, antioxidant enzymes and other biochemical attributes: Chlorophyll is described in the literature to be reduced under salinity stress in different crop plants like wheat (Mehta et al., 2010), pea (Ahmad and Jhon, 2005), rice (Anuradha and Rao, 2003) and tomato (Alaghabary et al., 2005; Zribi et al., 2009). Reactive oxygen species (ROS) are reported to be produced under stress conditions in different crop plants (Miller et al., 2010; Zhu, 2001). ROS damage the macro-molecules including chlorophyll and some proteins (Tambussi et al., 2000; Zhang et al., 2009). So this can be a solid reason for the deterioration of chlorophyll contents under salinity stress. MeJA is reported to counteract the negative effects of salinity on chlorophyll contents (Fedina et al., 2009; Kang et al., 2005). Under saline conditions proline contents also increased in different crop plants like pea (Velitchkova and Fedina, 1998), soybean (Yoon et al., 2009) and tomato (Claussen, 2005). MeJA application significantly increased free proline contents both under saline and non-saline conditions. Our findings are confirmed by the reports (Abdelgawad et al., 2014; Fedina and Benderliev, 2000; Velitchkova and Fedina, 1998), who depicted an increase in proline contents by MeJA application. Proline contents are reported to be increased under drought stress in pepper and also play a role against oxidative stress (Anjum et al., 2012). TSP and free amino acid contents were dramatically increased by the application MeJA under saline conditions but under normal conditions free amino acids gradually increased but TSP decreased. Our results are in line with Velitchkova and Fedina (1998) as they also recorded the same results in Pisum sativum. It is well documented (Abdelgawad et al., 2014; Fedina et al., 2009; Li et al., 1998; Popova et al., 2003) that MeJA augments antioxidants production under stress in different crop plants. In our results MeJA significantly enhanced the production of CAT and POX both under saline and non-saline conditions. Under stress conditions without foliar application of any chemical antioxidants are produced naturally against the response of oxidative stress of ROS (Gill and Tuteja, 2010). Thus in our study overproduction of CAT can be an adaptive mechanism of plants to stressful ecosystem (Nawaz et al., 2015) and MeJA has added in it. Plant species lacking the endogenous production of POX also fail in optimum membrane permeability due to lipid peroxidation, required to cope stress (Bhardwaj et al., 2009; Panda et al., 2003).

Conclusion: A significant increase was observed in enzymatic antioxidants and osmoprotectants by MeJA application under salt stressed tomato. It leads the plants to 
better withstand stress conditions by improved water relations and antioxidant system. A significant improvement in gaseous exchange parameters was also noted. There was a non-significant difference between 50 and $60 \mu \mathrm{M}$ MeJA application even in some cases $60 \mu \mathrm{M}$ performed better but if we optimize the best dose it remained $50 \mu \mathrm{M}$ MeJA foliar application for best recovery from salinity stress in tomato plants. Overall Rio Grande performed better than Savera.

\section{REFERENCES}

Abdelgawad, Z.A., A.A. Khalafaallah and M.M. Abdallah. 2014. Impact of methyl jasmonate on antioxidant activity and some biochemical aspects of maize plant grown under water stress condition. Agric. Sci. 5:10771088.

Ahmad, P. and R. Jhon. 2005. Effect of salt stress on growth and biochemical parameters of Pisum sativum L. (Einfluss Von Salzstress Auf Wachstum Und Biochemische Parameter Von Pisum Sativum L.). Arch. Agron. Soil Sci. 51:665-672.

Al-aghabary, K., Z. Zhu and Q. Shi. 2005. Influence of silicon supply on chlorophyll content, chlorophyll fluorescence, and antioxidative enzyme activities in tomato plants under salt stress. J. Plant Nutr. 27:21012115.

Anjum, S.A., L. Wang, M. Farooq, I. Khan, and L. Xue. 2011. Methyl jasmonate-induced alteration in lipid peroxidation, antioxidative defence system and yield in soybean under drought. J. Agron. Crop Sci. 197 :296301.

Anjum, S.A., M. Farooq, X. Xie, X. Liu and M.F. Ijaz. 2012. Antioxidant defense system and proline accumulation enables hot pepper to perform better under drought. Sci. Hortic. 140:66-73.

Anuradha, S. and S.S.R. Rao. 2003. Application of brassinosteroids to rice seeds (Oryza sativa L.) reduced the impact of salt stress on growth, prevented photosynthetic pigment loss and increased nitrate reductase activity. Plant Growth Regul. 40:29-32.

Aoki, A., A. Kanegami, M. Mihara, T. Kojima, M. Shiraiwa and H. Takahara. 2005. Molecular cloning and characterization of a novel soybean gene encoding a leucine-zipper-like protein induced to salt stress. Genet. 356:135-145.

Arnon, D.I. 1949. Copper enzymes in isolated chloroplasts. Polyphenoloxidase in Beta vulgaris. Plant Physiol. 24:115.

Bates, L.S., R.P. Waldren and I.D. Teare. 1973. Rapid determination of free proline for water-stress studies. Plant Soil. 39:205-207.

Bhardwaj, P., A.K. Chaturvedi and P. Prasad. 2009. Effect of enhanced lead and cadmium in soil on physiological and biochemical attributes of Phaseolus vulgaris. L. Nat. Sci. 7:63-75.

Chance, B. and A.C. Maehly. 1955. Assay of catalase and peroxidase. Method Enzymol. 2:764-775.

Claussen, W. 2005. Proline as a measure of stress in tomato plants. Plant Sci. 168:241-248.

Creelman, R.A. and R. Mulpuri. 2002. The oxylipin pathway in arabidopsis. The Arabidopsis book/American Society of Plant Biologists 1.

Cuartero, J. and R. Fernandez-Munoz. 1998. Tomato and salinity. Sci. Hortic. 78:83-125.

Davies, B. 1976. Carotenoids. In: T.W. Goodwin (ed.), Chemistry and biochemistry of plant pigments, $2^{\text {nd }} \mathrm{Ed}$. Academic Press, London. pp.38-165.

Del Amor, F.M. and P. Cuadra-Crespo. 2011. Alleviation of salinity stress in broccoli using foliar urea or methyljasmonate: Analysis of growth, gas exchange, and isotope composition. Plant Growth Regul. 63:55-62.

Fedina, I., D. Nedeva, K. Georgieva and M. Velitchkova. 2009. Methyl jasmonate counteract Uv-B Stress in barley seedlings. J. Agron. Crop Sci. 195:204-212.

Fedina, I.S. and K.M. Benderliev. 2000. Response of Scenedesmus incrassatulus to salt stress as affected by methyl jasmonate. Biol. Plant. 43 :625-627.

Flowers, T.J. and A.R. Yeo. 1995. Breeding for salinity resistance in crop plants: Where next? Funct. Plant Biol. 22:875-884.

Garcia-Mata, C. and L. Lamattina. 2002. Nitric oxide and abscisic acid cross talk in guard cells. Plant Physiol. 128:790-792.

Gill, S.S. and N. Tuteja. 2010. Reactive oxygen species and antioxidant machinery in abiotic stress tolerance in crop plants. Plant Physiol. Biochem. 48:909-930.

Hamilton, P.B. and D.D. Van Slyke. 1943. Aminoacids determination with ninhydrin. J. Biol. Chem. 150:231233.

Jia, W., Y. Wang, S. Zhang and J. Zhang. 2002. Salt-stress-induced aba accumulation is more sensitively triggered in roots than in shoots. J. Exp. Bot. 53:22012206.

Jin, S., C.C.S. Chen and A.L. Plant. 2000. Regulation by Aba of osmotic-stress-induced changes in protein synthesis in tomato roots. Plant Cell Environ. 23:51-60.

Kang, D.J., Y.J. Seo, J.D. Lee, R. Ishii, K.U. Kim, D.H. Shin, S.K. Park, S.W. Jang and I.J. Lee. 2005. Jasmonic acid differentially affects growth, ion uptake and abscisic acid concentration in salt-tolerant and salt-sensitive rice cultivars. J. Agron. Crop Sci. 191:273-282.

Li, L., J.V. Staden and A.K. Jäger. 1998. Effects of plant growth regulators on the antioxidant system in seedlings of two maize cultivars subjected to water stress. Plant Growth Regul. 25:81-87. 
Lowry, O.H., N.J. Rosebrough, A.L. Farr and R.J. Randall. 1951. Protein measurement with folin phenol reagent. J. Biol. Chem. 191:265-275.

Lutts, S., J.M. Kinet and J. Bouharmont. 1996. Effects of salt stress on growth, mineral nutrition and proline accumulation in relation to osmotic adjustment in rice (Oryza Sativa L.) cultivars differing in salinity resistance. Plant Growth Regul. 19:207-218.

Maggio, A., G. Raimondi, A. Martino and S. De Pascale. 2007. Salt stress response in tomato beyond the salinity tolerance threshold. Environ. Exp. Bot. 59:276-282.

Mehta, P., A. Jajoo, S. Mathur and S. Bharti. 2010. Chlorophyll a fluorescence study revealing effects of high salt stress on Photosystem II in wheat leaves. Plant Physiol. Biochem. 48:16-20.

Miller, G.A.D., N. Suzuki, S. Ciftci-Yilmaz and R. Mittler. 2010. Reactive oxygen species homeostasis and signalling during drought and salinity stresses. Plant Cell Environ. 33:453-467.

Munns, R. and M. Tester. 2008. Mechanisms of salinity tolerance. Annu. Rev. Plant Biol. 59:651-681.

Nawaz, F., M.Y. Ashraf, R. Ahmad, E.A. Waraich, R.N. Shabbir and M.A. Bukhari. 2015. Supplemental selenium improves wheat grain yield and quality through alterations in biochemical processes under normal and water deficit conditions. Food Chem. 175:350-357.

Panda, S.K., I. Chaudhury and M.H. Khan. 2003. Heavy metals induce lipid peroxidation and affect antioxidants in wheat leaves. Biol. Plant. 46:289-294.

Poor, P., K. Gemes, F. Horvath, A. Szepesi, M.L. Simon and I. Tari. 2011. Salicylic acid treatment via the rooting medium interferes with stomatal response, $\mathrm{CO}_{2}$ fixation rate and carbohydrate metabolism in tomato, and decreases harmful effects of subsequent salt stress. Plant Biol. 13:105-114.

Popova, L., E. Ananieva, V. Hristova, K. Christov, K. Georgieva, V. Alexieva and Z.H. Stoinova. 2003. Salicylic acid and methyl jasmonate-induced protection on photosynthesis to paraquat oxidative stress. Bulg. J. Plant Physiol. Special Issue 133-152.
Romero-Aranda, R., T. Soria and J. Cuartero. 2001. Tomato plant-water uptake and plant-water relationships under saline growth conditions. Plant Sci. 160:265-272.

jasmonate and the role of calcium and abscisic acid in this process. Plant Cell Environ. 37:995-1008.

Suhita, D., V. A. Kolla, A. Vavasseur and A.S. Raghavendra. 2003. Different signaling pathways involved during the suppression of stomatal opening by methyl jasmonate or abscisic acid. Plant Sci. 164:481488.

Tambussi, E.A., C.G. Bartoli, J. Beltrano, J.J. Guiamet and J.L. Araus. 2000. Oxidative damage to thylakoid proteins in water-stressed leaves of wheat (Triticum Aestivum). Physiol. Plant. 108:398-404.

Tsonev, T.D., G.N. Lazova, Z.H.G. Stoinova and L.P. Popova. 1998. A possible role for jasmonic acid in adaptation of barley seedlings to salinity stress. J. Plant Growth Regul. 17:153-159.

Velitchkova, M. and I. Fedina. 1998. Response of photosynthesis of Pisum sativum to salt stress as affected by methyl jasmonate. Photosynthetica 35:8997.

Wang, S.Y. 1999. Methyl jasmonate reduces water stress in strawberry. J. Plant Growth Regul. 18:127-134.

Wasternack, C. and B. Hause. 2002. Jasmonates and octadecanoids: Signals in plant stress responses and development. Prog. Nucleic acid Res. 72:165-221.

Yoon, J.Y., M. Hamayun, S. Lee and I. Lee. 2009. Methyl jasmonate alleviated salinity stress in soybean. J. Crop Sci. Biotechnol. 12:63-68.

Zhang, H., Y. Ye, S. Wang, J. Luo, J. Tang and D. Ma. 2009. Hydrogen sulfide counteracts chlorophyll loss in sweet potato seedling leaves and alleviates oxidative damage against osmotic stress. Plant Growth Regul. 58:243-250.

Zhu, J. 2001. Plant salt tolerance. Trends Plant Sci. 6:66-71.

Zribi, L., G. Fatma, R. Fatma, R. Salwa, N. Hassan and R.M. Nejib. 2009. Application of chlorophyll fluorescence for the diagnosis of salt stress in tomato 'Solanum Lycopersicum (Variety Rio Grande)'. Sci. Hortic. 120:367-372. 